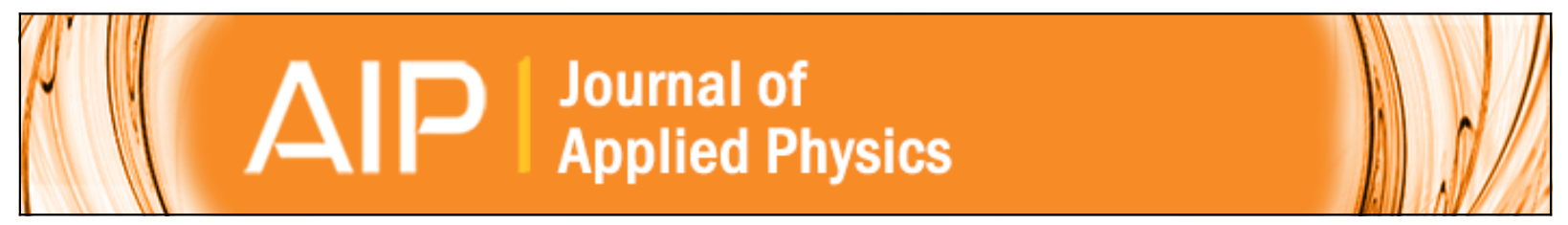

\title{
Barrier Energies in MIM Structures from Photoresponse: Effect of Scattering in the Insulating Film
}

G. Lewicki, J. Maserjian, and C. A. Mead

Citation: Journal of Applied Physics 43, 1764 (1972); doi: 10.1063/1.1661392

View online: http://dx.doi.org/10.1063/1.1661392

View Table of Contents: http://scitation.aip.org/content/aip/journal/jap/43/4?ver=pdfcov

Published by the AIP Publishing

\section{Articles you may be interested in}

Nanomanufacturability Of Thin Film MIM Diodes

AIP Conf. Proc. 1313, 403 (2010); 10.1063/1.3530561

Effect of an inhomogeneous insulating film on the capacitance of metal-insulator-semiconductor structures J. Appl. Phys. 84, 3960 (1998); 10.1063/1.368574

Photoresponse of semiconductor on semiinsulator structure

J. Appl. Phys. 75, 5321 (1994); 10.1063/1.355734

ac equivalent circuits for MIM structures

J. Appl. Phys. 45, 2907 (1974); 10.1063/1.1663699

ac properties of biased MIM structures

J. Appl. Phys. 45, 1223 (1974); 10.1063/1.1663393

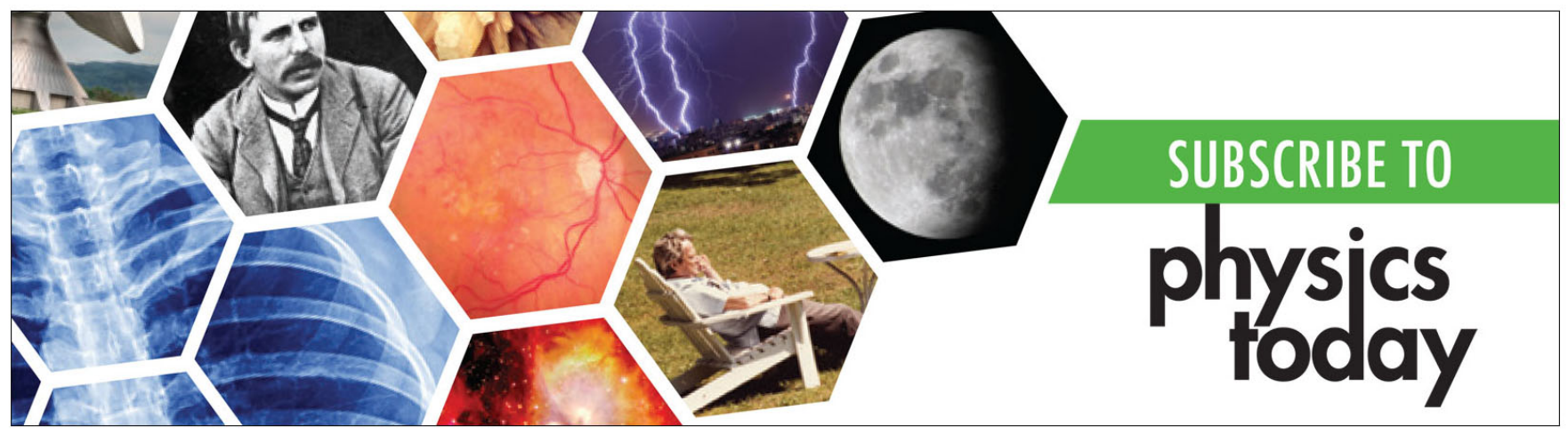




\title{
Barrier Energies in MIM Structures from Photoresponse: Effect of Scattering in the Insulating Film*
}

\author{
G. Lewicki and J. Maserjian \\ Jet Propulsion Laboratory, Califormia Institute of Technology, Pasadena, Califormia 91103 \\ and \\ C. A. Mead \\ California Institute of Technology, Pasadena, Califormia 91109 \\ (Received 24 September 1971)
}

\begin{abstract}
Scattering of electrons photoexcited into the insulator conduction band prevents photoresponse from following the Fowler relation in MIM structures. However, barrier energies can be obtained without specific knowledge of the scattering process either by measuring the threshold for photoresponse directly, or by applying sufficiently large voltages across the insulator.
\end{abstract}

\section{INTRODUCTION}

Definition of current-flow mechanisms in MIM (metalinsulator-metal) structures requires knowledge of barrier energies at the metal-insulator interfaces. Photoresponse measurements are considered a convenient means of determining these barrier energies. Nevertheless, such measurements on $\mathrm{Al}-\mathrm{Al}_{2} \mathrm{O}_{3}-M^{1}$ and Al-AlN$\mathrm{Mg}^{2}$ structures interpreted in terms of the Fowler relation $^{3}$ led to barrier energies dependent on insulator thickness and applied voltage in a manner inconsistent with a trapezoidal barrier modified by image forces. This left some doubt as to the validity of the barrier energies deduced in this manner.

To explain the photoresponse data on Al-AlN-Mg structures presented by two of the authors, ${ }^{2}$ Simmons proposed a model in which negative ionic space charge within the insulator appreciably distorts the barrier from a trapezoidal shape. ${ }^{4}$ He showed that the photoresponse data could be explained with reasonable values being given to the various parameters of his model.

Schuermayer et al. ${ }^{5}$ observed deviations from the Fowler relation in $\mathrm{Al}-\mathrm{Al}_{2} \mathrm{O}_{3}-\mathrm{Au}$ structures and attempted to explain them in terms of a specific model of scattering of electrons photoexcited into the insulator conduction band.

It is the purpose of this letter to present new data to show (i) that the barrier shape in Al-AlN-M structures ${ }^{6}$ in the insulator-thickness range $30 \AA$ to at least $50 \AA$ is nearly trapezoidal and not appreciably altered by ionic space charge; (ii) that deviations from the Fowler relation are observed in Al-AlN- $M$ structures and that these deviations can be unambiguously interpreted in terms of scattering of electrons photoexcited into the conduction band of AlN without assuming any specific details of the scattering process; and (iii) how barrier energies can be determined directly and accurately from photoresponse measurements in the presence of scattering again without specific knowledge of the scattering process.

\section{THEORETICAL CONSIDERATIONS}

The photocurrent $I$ resulting from photoexcitation of electrons within one metal, these electrons passing to the other metal through the conduction band of the insulator, is usually described by the low-temperature approximation of the Fowler relation, which has been proved experimentally valid in many metal-semiconductor structures:

$$
Y=\frac{I}{e F} \approx \begin{cases}a(h \nu-\phi)^{2} & \text { for } h \nu>\phi \\ 0 & \text { for } h \nu<\phi,\end{cases}
$$

where $Y$ is the photoyield, $e$ is the electronic charge, $F$ is the incident photon flux, $a$ is a constant describing the efficiency of the photoexcitation process, $h \nu$ is the photon energy, and $\phi$ is the barrier energy. The usual method for photoelectrically determining barrier energies involves plotting measured values of $Y^{1 / 2}$ as a function of $h \nu$, fitting a straight line to the data, and extrapolating this line to the photon energy for which $Y^{1 / 2}=0$. According to Eq. (1), this energy is the barrier energy.

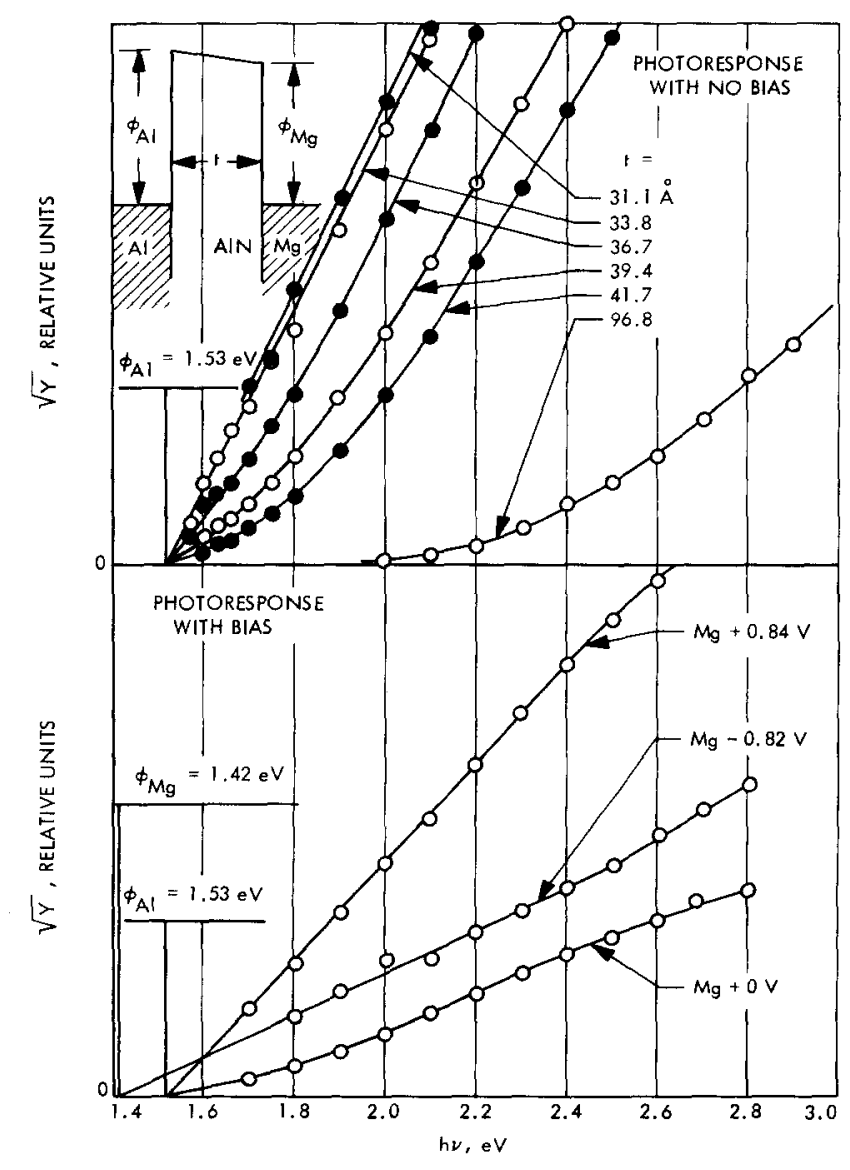

FIG. 1. Photoresponse in Al-AlN-Mg structures. 


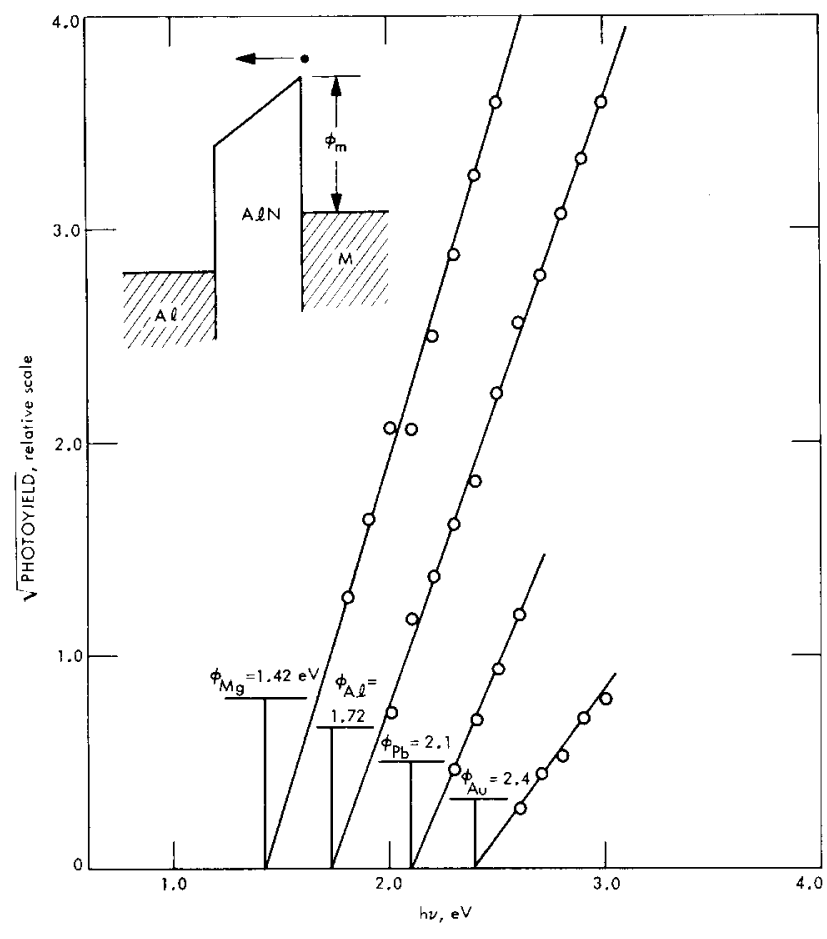

FIG. 2. Saturated photoresponse in Al-AlN- $M$ structures.

The Fowler relationship implicitly assumes unity transmission through the insulator conduction band for all electrons photoexcited into it. If there is significant scattering of electrons within the insulator conduction band, the transmission $T$ of an electron photoexcited to an energy $h \nu>\phi$ above the Fermi level of the metal in which the excitation process is taking place is not unity but a complicated function of $h \nu$ and field within the insulator. This function is, in general, unknown and can be deduced only so far as the details of the scattering mechanism are known. ${ }^{5}$

In the case of scattering, Eq. (1) is not valid and the photoresponse can-with arguments similar to those used in deriving the Fowler relationship-be related to $T(h \nu)$ by

$$
\begin{aligned}
\frac{1}{2 a} \frac{\partial^{2} Y}{\partial(h \nu)^{2}} & =T(h \nu) & & \text { for } h \nu>\phi \\
Y & \approx 0 & & \text { for } h \nu<\phi .
\end{aligned}
$$

For the special case where $T(h \nu)=1$ (i.e., no scattering), Eqs. (1) and (2) are equivalent. In the case of scattering, a barrier energy cannot be extrapolated on the basis of Eq. (1) and without knowledge of $T(h \nu)$ can be determined only by direct measurement of the photon energy at which the photoyield falls to zero.

\section{RESULTS AND DISCUSSION}

In the top half of Fig. 1 are shown room-temperature plots of $Y^{1 / 2}$ as a function of photon energy $h \nu$ in Al-AlN$\mathrm{Mg}$ structures with insulator thicknesses ranging from 31 to $97 \AA$, as estimated from their capacitance. The measurements were taken with a double-pass grating spectrometer with the structures illuminated through highly transparent aluminum-base electrodes $(\approx 40 \AA$ thick). The thin-base samples gave relatively large photoyields which, when combined with a high-intensity xenon lamp and a sensitive phase-lock amplifier, allowed measurements to be made very close to the true threshold.

From their polarity, we know the photocurrents to be mainly the result of electrons photoexcited in the base Al electrode. We also know that with no voltage applied to the Mg electrode, there is a slight built-in field that assists propagation of these photoexcited electrons through the insulator conduction band (see inset in the top half of Fig. 1).

Only in the structures with the thinnest insulators is the Fowler relation observed. As the insulator thickness increases, the photoyield has an intercept at the same photon threshold energy (within experimental uncertainty) but does not give a straight line in a plot of $Y^{1 / 2}$ vs $h \nu$ over the whole range of data. From this and other similar data, we can say that the barrier energy at the $\mathrm{Al}-\mathrm{AlN}$ interface is $1.53 \mathrm{eV}$ and that it is independent of insulator thickness in the range $30 \AA$ to at least $50 \AA$. In this range of insulator thickness, negative space charge does not appeciably alter the barrier.

The slopes of the curves shown in the top half of Fig. 1 are a rough measure of the second derivative of $Y$ with respect to $h \nu$, or $T(h \nu)$ as defined in Eq. (2). Consequently the data tell us that, as generally expected from scattering, the transmission of photoexcited electrons decreases with increasing insulator thickness, this decrease being more pronounced for energies near the barrier energy than for higher energies.

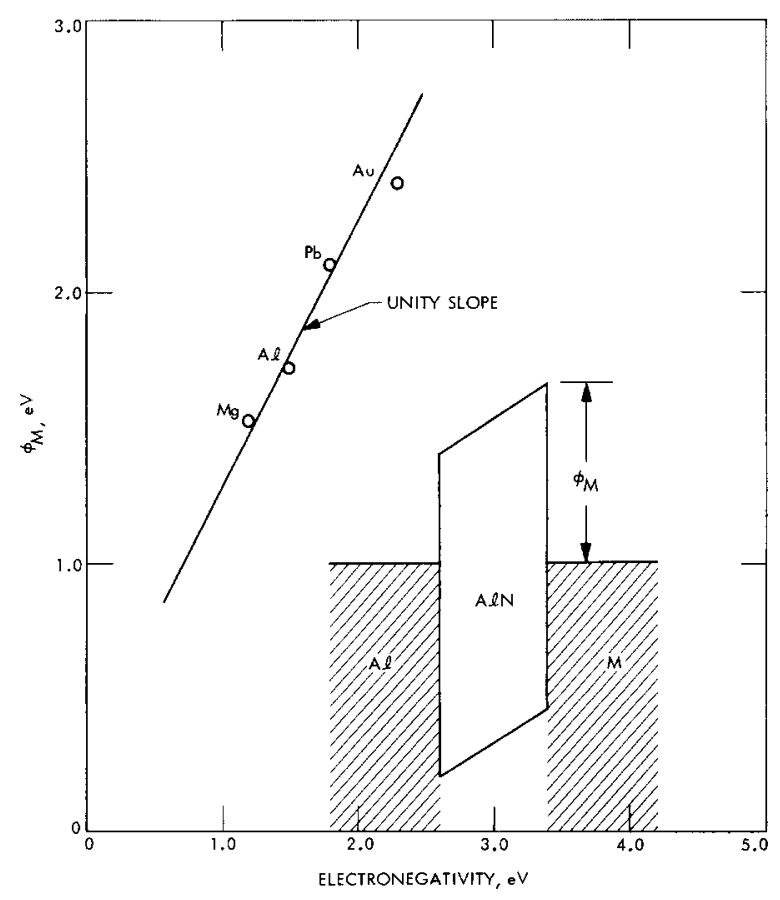

FIG. 3. Barrier energies versus electronegativity. 


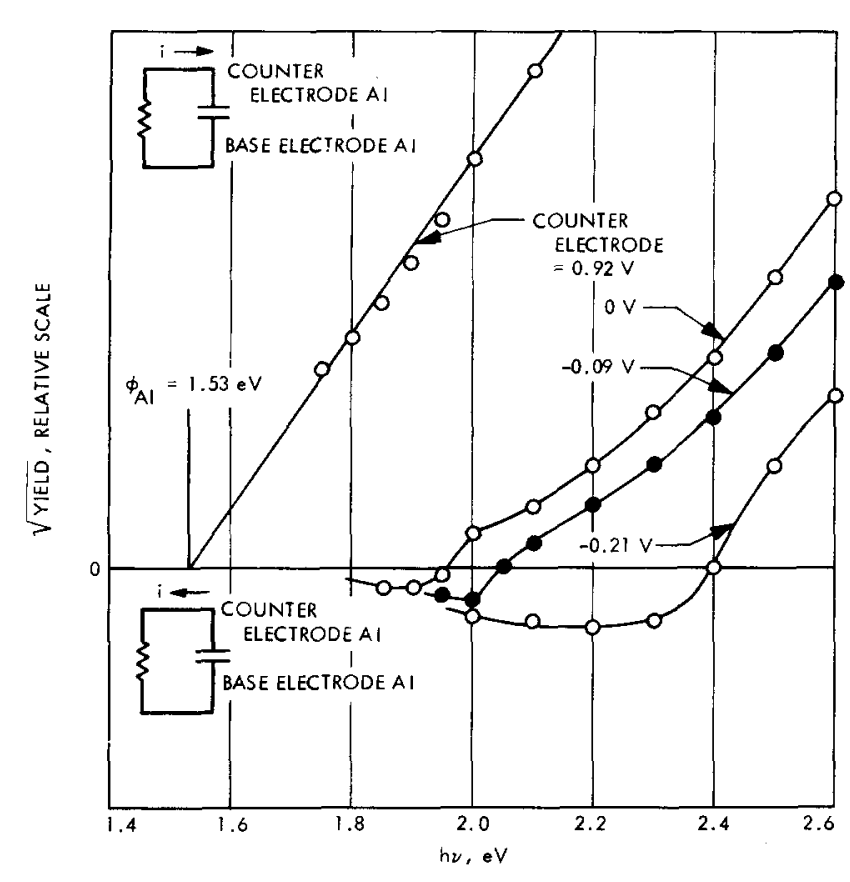

FIG. 4. Photoresponse in Al-AIN-Al structures. In the top half of the graph are plotted $|Y|^{1 / 2}$ for photocurrents with polarities corresponding to photcemission of electrons from the base electrode; in the bottom half $|Y|^{1 / 2}$ for photoemission of electrons from the counterelectrode.

Another method of determining barrier energies directly from photoresponse, in the case of appreciable scattering, involves the application of sufficiently large voltages across the structures.

In the bottom half of Fig. 1 are shown plots of $|Y|^{1 / 2}$ vs $h \nu$ at $77^{\circ} \mathrm{K}$ for an Al-AlN-Mg structure with an intermediate insulator thickness with various voltages applied to the $\mathrm{Mg}$ electrode. With no applied voltage, a deviation from the Fowler relation is obtained. As a positive voltage is applied to the Mg electrode, the photoyield increases and eventually saturates in the sense that further increases in voltage do not increase the photoyield. ${ }^{7} \mathrm{~A}$ saturated photoresponse does follow the Fowler relation in that a plot of $Y^{1 / 2}$ vs $h \nu$ does yield a straight line. Such a saturated response is shown for $0.84 \mathrm{~V}$ applied to the $\mathrm{Mg}$ electrode. At this voltage the field is sufficiently large to sweep through the insulator conduction band all electrons photoexcited into it. The extrapolated photon energy threshold for the photoresponse shown unambiguously defines the electron barrier energy $\phi_{A 1}$ at the Al-AlN interface. This barrier energy agrees with that obtained with no voltage across structures with thicker and thinner insulators.

As a negative voltage is applied to the $\mathrm{Mg}$ electrode, the polarity of the photocurrent reverses, indicating photoemission of electrons from the Mg electrode. For sufficiently large negative voltages, the photoresponse again saturates. Such a saturated response is shown in the bottom half of Fig. 1 for $-0.82 \mathrm{~V}$ applied to the $\mathrm{Mg}$ electrode. The extrapolated photon energy threshold for this response unambiguously defines the electron barrier energy $\phi_{\mathrm{Mg}}$ at the AlN-Mg interface. It is to be noted from the magnitudes of the responses that the photoex- citation process is more efficient in the base Al electrode than in the $\mathrm{Mg}$ counterelectrode.

Barrier energies obtained from saturated photoresponses in structures $\mathrm{Al}-\mathrm{AlN}-\mathrm{Al},-\mathrm{Pb},-\mathrm{Au}$ were also 1.53 $\mathrm{eV}$ at the base aluminum insulator interface and from the data plotted in Fig. 2, $1.72 \mathrm{eV}, 2.1 \mathrm{eV}$, and $2.4 \mathrm{eV}$ at the insulator-aluminum, -lead, and -gold interfaces, respectively. The barrier energies at the AIN- $M$ interfaces can be seen in Fig. 3 to vary almost directly with the electronegativity of the metal. Photoresponse measurements we have made on $\mathrm{Al}-\mathrm{Al}_{2} \mathrm{O}_{3}-M$ structures yielded barrier energies lower than with $\mathrm{Al}-\mathrm{AlN}-M$ structures.

The deviation from the Fowler relationship cannot be explained on the basis of tunnel penetration of electrons photoexcited to energies below the barrier energy. If this were the case, for zero voltage applied across a structure, the total photocurrent resulting from photoexcitation of electrons within both electrodes would have the same polarity for all values of $h \nu$; that is, the transmisstion of electrons excited in the two electrodes would have the same dependence on $h \nu$. Experimentally, this is not the case.

In Fig. 4 are shown photoresponse data for an Al-AlN$\mathrm{Al}$ structure with various voltages applied to the $\mathrm{Al}$ counterelectrode. The barrier energy at the AlN-Al interface is larger than that at the Al-AlN interface and thus there is a built-in field within this structure opposite in direction to that within $\mathrm{AlN}-\mathrm{Mg}$ structures. The graph is plotted such that positive values of $|Y|^{1 / 2}$ represent photocurrents with polarities corresponding to photoemission of electrons from the base electrode, and negative values of $|Y|^{1 / 2}$ represent photocur rents with polarities corresponding to photoemission of electrons from the counterelectrode.

With no applied voltage, the photocurrent changes polarity as $h \nu$ is varied. This result is expected on the basis of scattering. More electrons are being photoexcited in the base $\mathrm{Al}$ electrode than in the $\mathrm{Al}$ counterelectrode because the structure is illuminated through the base electrode. But electrons photoexcited in the base electrode must propagate through the insulator conduction band against a built-in field, while those excited in the counterelectrode propagate with the aid of the builtin field. Thus, for low values of $h \nu$, the flow of electrons from the counterelectrode to the base electrode predominates. On the other hand, the transmission of electrons photoexcited to high energies is relatively independent of the polarity of the field. Consequently, at larger values of $h \nu$, the flow of electrons from the base electrode to the counterelectrode predominates.

The data plotted in Fig. 4 clearly show that the total photocurrent for zero applied bias cannot be described as the sum of two photocurrents due to photoemission of electrons from the two Al electrodes, with each photocurrent having the same dependence on $h \nu$.

\section{CONCLUSIONS}

Backward extrapolation of data taken at high values of $h \nu$ to obtain threshold energies for photocurrents on the basis of the Fowler relation is a valid procedure only in 
special circumstances. The insulator must be sufficiently thin, and there must be a field within the insulator large enough to sweep through the insulator conduction band all electrons photoexcited into it. The variation of the apparent barrier energy with insulator thickness and applied voltage reported previously on Al-AlNMg structures was a misinterpretation of data in that the photocurrent was assumed to follow the Fowler relation when, in fact, it did not. The threshold energies deduced are not barrier energies. Straight lines were drawn through photoresponse data such as those shown in the top half of Fig. 1, ignoring lower photon energy points which could not be resolved with the experimental apparatus used at that time. The data in the top half of Fig. 1 show that the threshold energy for photoresponse in $\mathrm{Al}-\mathrm{AlN}-\mathrm{Mg}$ structures is independent of insulator thickness at least in the range $31-42 \AA$, if not to $97 \AA$; other data, not shown, increase the certainty of the upper limit to $50 \AA$. This fact gives assurance that within this thickness range there is insufficient space charge in the insulator to distort the barrier appreciably from a trapezoidal shape. This conclusion is in agreement with studies of tunneling currents in the $\mathrm{Al}-\mathrm{AlN}-M$ structures. ${ }^{8}$

*This paper presents the results of work performed with the California Institute of Technology under a work order with the Jet Propulsion Laboratory. Sponsored by the National Aeronautics and Space Administration under Contract NAS 7-100.

${ }^{1}$ A.I. Braunstein, M. Braunstein, and J.S. Picus, Appl. Phys. Letters 8, 95 (1966).

${ }^{2}$ G. W. Lewicki and C.A. Mead, Appl. Phys. Letters 8, 98 (1966).

${ }^{3}$ R. H. Fowler, Phys. Rev, 38, 45 (1931)

${ }^{4}$ J. G. Simmons, Phys. Rev. Letters 23, 297 (1969).

${ }^{5}$ F. L. Schuermeyer, C.R. Young, and J.M. Blasingame, J. Appl. Phys. 39, 1791 (1968).

${ }^{6}$ The designation AlN for the insulating films used in this work is strictly a convenient measure, since at this time we lack precise information on the film composition. The films were formed in a gas discharge containing primarily nitrogen and traces of oxygen.

7An anomalous "bump" in excess of the apparent saturated response appears at low photon energies for large applied fields. This phenomenon is presently under investigation.

${ }^{8}$ G. Lewicki and C.A. Mead, Phys. Rev. Letters 16, 939 (1966).

\title{
Analysis of Doping Anomalies in GaAs by Means of a Silicon-Oxygen Complex Model
}

\author{
M. E. Weiner and A.S. Jordan \\ Bell Telephone Laboratories, Murray Hill, New Jersey 07974 \\ (Received 29 July 1971; in final form 16 November 1971)
}

\begin{abstract}
A model based on the formation of silicon-oxygen pairs is proposed to explain a variety of anomalous phenomena associated with GaAs grown in the presence of silicon, $\mathrm{SiO}_{2}$, and/or oxygen. It is suggested that silicon atoms on gallium sites pair with interstitial oxygen atoms, forming a complex which behaves as an acceptor with energy levels near 0.2 and $0.4 \mathrm{eV}$ below the conduction band. It is assumed that the complex can dissociate upon annealing below $850^{\circ} \mathrm{C}$ by the reaction $2\left(\mathrm{Si}_{\mathrm{Ga}_{2}} \mathrm{O}\right)^{-}=\left(\mathrm{Si}_{\mathrm{Ga}_{2}} \mathrm{O}_{2}\right)^{0}+\mathrm{Si}_{\mathrm{Ga}}^{+}+3 e^{-}$. This reaction may be reversed at higher temperatures. The electrical and thermochemical properties of such a complex can explain annealing behavior, the occurence of a mobility maximum between 150 and $200^{\circ} \mathrm{K}$, site distribution of silicon in $p$-type silicon-doped GaAs, change from $n$ - to $p-$ type conductivity as a function of Si concentration in GaAs grown from Ga solutions, the formation of $I$ (insulating) layers in GaAs grown by vapor-phase epitaxy, and some of the anomalous behavior observed in GaAs devices. A variety of growth experiments and characterization techniques which could aid in verification of the $\mathrm{Si}_{\mathrm{Ga}_{a}} \mathrm{O}_{i}$ complex model are suggested.
\end{abstract}

\section{INTRODUCTION}

Silicon is a widely used dopant for GaAs. In melt growth, it is an $n$-type dopant and is often used in the growth of crystals for degenerate substrates. In liquidphase epitaxial growth, Si may be either an $n$-type dopant or $p$-type dopant, depending on temperature, concentration, and growth rate. Thus the growth of amphoteric $p-n$ junctions in GaAs is possible. ${ }^{1,2}$ Silicon is also expected to be a common contaminant in GaAs because of various chemical reactions with the fused quartz which is commonly used in various growth systems. ${ }^{3}$ There are a number of anomalies observed in both intentionally doped and silicon-contaminated GaAs which appear to be correlated with the presence of oxygen as an additional contaminant. It is the purpose of this paper to propose a model which can account, at least qualitatively, for a variety of anomalous effects observed in GaAs contaminated or doped with $\mathrm{Si}$.

\section{ANOMALOUS PHENOMENA IN GaAs ASSOCIATED WITH THE PRESENCE OF Si}

\section{A. Anomalous Energy Levels}

An energy level near $0.2 \mathrm{eV}$ has been observed in $n$-type GaAs grown by the sealed-tube Bridgman technique from a stoichiometric melt in a quartz boat with a small amount of oxygen added to the ampoule. ${ }^{4}$ The energy of this level was obtained from the temperature variation of the Hall voltage. An energy level of $0.158 \mathrm{eV}$ was observed by Basinski ${ }^{5}$ in similar material. He fitted his Hall data by assuming the level to be a deep donor. A level close to $0.2 \mathrm{eV}$ has been observed in photoluminescence measurements on liquid-phase epitaxial (LPE) layers of GaAs grown in quartz ${ }^{6}$ and in low-frequency noise measurements on Gunn devices fabricated from open-tube vapor-phase epitaxial (VPE) layers of GaAs. ${ }^{7}$ 